\title{
A care counselling model for HIV reactive patients in rural Malawi - Part II
}

\author{
Y Sliep, D.Cur(Student), Department of Nursing, RAU \\ M Poggenpoel, Ph.D, Department of Nursing, RAU \\ A Gmeiner, D.Cur, Department of Nursing, RAU
}

\section{Abstract}

The purpose of the research conducted was to explore and describe HIV patients experience of their illness within the context of rural Malawi and, based on the results of the study, to describe a model for counselling HIV reactive patients in rural Malawi which would meet both the internal and external needs of the patient.

Nursing for the Whole Person Theory, as well as symbolic interactionism and the research model of Botes, were used as paradigm, as the researcher believes a person is fundamentally a historical social being who can only be understood in a specific cultural context of space and time. The research was also conducted within a counselling context and different disciplines were integrated in the study.

The research method followed two phases each addressing a different objective. Firstly, exploration and description of the HIV reactive individual's experience of illness, compilation of a demographic profile of the patients in the study and a description of their identified needs as well as possible resources, exploration and description of the primary care givers' experience of HIV reactive patients and the exploration and description of the viewpoint of counsellors in terms of counselling HIV reactive patients.

In the first phase of the study in-depth phenomenological interviews were conducted with identified groups. Focus interviews were conducted with a hundred AIDS patients to identify the needs and resources of the patients and to compile a demographic profile. Focus groups discussions were conducted with counsellors for more complete comprehension. Data-analysis and literature control was undertaken.

In the second phase of the study theory generation was used in order to develop a counselling model for AIDS patients and guidelines for implementing the model were generated. Based on the results of the analysis the major concept enable was identified as the essence of a model for counselling AIDS patients in rural Malawi.

The visual model developed described a process of enablement with the counsellor as negotiator and the patient as a narrator in which the patient, family and community are encouraged to participate actively. By utilising the deductive reasoning strategy, relationship statements were inferred from the model. Guidelines were described for all three phases of the model, namely pretest counselling, post-test counselling and community care, in terms of objectives, strategies and activities.

The value of this research was embodied in addressing a very real and urgent need in Malawi and proposing a solution with practical guidelines.

\section{Overview and problem statement}

In Malawi Human Immunodeficiency Virus (HIV)/ Acquired Immune Deficiency Syndrome (AIDS) has become one more problem amongst many others facing the continent of Africa. The HIV infection is a major and rapidly growing health problem that is not limited to a small high-risk segment of the population. The first seven cases in Malawi were reported in 1985. Since then, according to figures supplied by the AIDS Secretariat of the Ministry of Health (AIDSEC), the number of cases has grown steeply. The prevalence of HIV/AIDS resembles that of highly endemic countries in Africa. By June 1994 a cumulative total of 34866 AIDS cases had been reported and cumulative total of $875096 \mathrm{HIV}$ cases were reported. Of these $92 \%$ were adults and $4 \%$ children below five years. This HIV prevalence is estimated at $23 \%$ in urban areas and $8 \%$ in rural areas is one of the highest in the world. Studies of antenatal mothers in Lilongwe and Blantyre confirm a sharp rise of HIV infection, from 8,2\% in 1985 to about 32\% in 1994 (Malawi AIDS Control Programme, 1994:1).

The high incidence rate of HIV/AIDS in Malawi has had a big impact on the health services in Malawi. The number of patients has increased and health services have become overstretched. Counselling services, before the endemic a fairly unknown concept within the health sector, are now recognised as an important component of the health care provided for HIV/AIDS patients - although the concept is still unknown to patients, especially in rural areas.

According to the WHO counselling model that has been adopted by Malawi, counselling is described as a dynamic interactional process between an individual (a counsellor) and 
another individual (a client), or group of individuals. HIVrelated counselling is seen as a goal-orientated activity aimed firstly at prevention of spreading the virus and secondly at optimising personal well being (AIDS Control Programme, 1992: $19-25)$.

Every patient who is tested for HIV has to be pre-test and posttest counselled. Follow-up counselling is recommend but rarely done (Sliep, 1994: 9).

A number of problems were encountered during the implementation of this model. The first one was an inappropriate selection of counsellors. It is not clear what training counsellors should have, neither what the qualities and expectations of a counsellor should be.

Secondly the motivation of the counsellors who underwent counselling training could be questioned. It is standard practice in Malawi that people attending workshops are paid money in addition to their salary. Paying of additional allowances has resulted in people attending training workshops for the money instead of for the knowledge (Sliep, 1994: 10).

Another problem that had not been foreseen was the inability of the hospital to accommodate counselling on a large scale. Sufficient time is needed and this often brings a considerable workload. A private area where counselling can be conducted, is often lacking.

The counselling model which that chosen has a pre-condition that both the counsellor and the patient should have the ability to think critically. It is assumed that the participant will be able to apply the problem solving method proposed by the programme and that their patient will be able to participate in that process. Considering the extremely low level of education in Malawi, it is unlikely that patients will be able to participate in problem solving, even if given guidance (Sliep, 1994: 11).

Unlike the situation in the West, testing for HIV is not a wellknown or accepted concept. The majority of the population still not often requests testing, as there is a fear and stigma about attached to the illness that prevents testing. Testing is also not readily available every where and means an additional expense for the patient.

Lastly, the present model is individual orientated that could cause alienation from the group (family and community) to which the patient belongs. The African context is not considered sufficiently in the person centred counselling approach. For the African person belonging forms an integral and essential part of well being and therefore needs to be integrated into the counselling approach.

During the year prior to the research being conducted, the researcher became aware of the fact that the patients did not feel that they benefited from being counselled about HIV/AIDS issues. Counsellors felt frustrated in their work and saw themselves as messengers of death. This observation made by the researcher was confirmed by the AIDS Secretariat during an evaluation done on the effectiveness of counselling.

Based on the above, the following research question was asked: What approach to counselling would be specific in meeting the needs of HIV/AIDS patients within a family and a community context in a rural area in Malawi?

\section{Purpose and conceptual framework}

The aim of the research was to describe a model of counselling HIV reactive patients that would meet the needs of such a patient in a rural African community in Malawi. To achieve the aim of the research, the following objectives were set:

1. To explore and describe an approach to counselling that would facilitate the needs and strengths of the HIV/AIDS patients within a family and community context in a rural area in Malawi.

- Explore and describe the HIV reactive individuals' experience of illness.

- $\quad$ Compile a demographic profile of the patient in the study and describe who the patient identifies as possible resources and why.

- $\quad$ Explore and describe the experience of care givers of an AIDS patient.

- $\quad$ Explore and describe the viewpoint of counsellors in terms of counselling HIV reactive patients.

2. To develop a counselling model based on the findings of the research study that will meet the needs of AIDS patients within a community context in rural Malawi.

The Central theoretical statement of the research read as follows:

Insight into the experience of the AIDS patient, care giver and counsellor provides the basis for a counselling model that will strengthen the individual patient within his/her family and community.

The research was mainly conducted within a counselling context and different disciplines and paradigms are integrated in the study. The Department of Nursing at the Rand Afrikaans University has accepted the Nursing for the Whole Person Theory of the Oral Roberts University Anna Vaughn School of Nursing (1990: $136-139 ; 142$ ), which is congruent with the philosophy of the Rand Afrikaans University (Department of Nursing, Rand Afrikaans University, 1991: 1).

For the purpose of this research study the Nursing of the Whole Person Theory was also used, but the researcher did not accept the metatheoretical assumptions of the theory, which are based on a Judeo-Christian perspective. The researcher believes a person is fundamentally a historical social being who can only be understood in a specific cultural context of space and time.

The theory reflects the focus on the whole person - body, mind and spirit where:

mind = emotional, volitional and intellectual processes

body $=$ physiological (biological) processes

spirit = that part of man created for communion with God

The researcher would like to rephrase the term spirit as the core of an individual's existence, integrating and transcending the physical, emotional, intellectual and social dimensions (Sliep, 


\begin{tabular}{|c|c|c|}
\hline & PROCESS & METHODOLOGY \\
\hline Phase I & $\begin{array}{l}\text { - Exploration and description of an } \\
\text { approach to counselling of the HIV/ } \\
\text { AIDS patient }\end{array}$ & $\begin{array}{ll}\text { - } & \text { Purposive sampling according to criteria } \\
\text { - } & \text { Demographic data } \\
\text { - } & \text { counso focus group discussions with all trained } \\
\text { - } & \text { In-depth interviews with HIV reactive Malawi’s } \\
\text { - } & \text { Data-analysis (Kerlinger, 1986: } 477-480 \text { ) } \\
\text { - } & \text { Conclusions }\end{array}$ \\
\hline \multirow[t]{3}{*}{ Phase II } & $\begin{array}{l}\text { Exploration and description of con- } \\
\text { cepts relevant to the mode. (Theory } \\
\text { generation design according to } \\
\text { Chinn \& Kramer 1991: 79-145) }\end{array}$ & $\begin{array}{l}\text { - } \quad \text { Results from Phase I used to identify concepts } \\
\text { - } \quad \text { Dictionary and subject definitions } \\
\text { Constructing of a model case of concept (Chinn } \\
\text { \& Kramer, 1991: 79- 105) }\end{array}$ \\
\hline & $\begin{array}{l}\text { Generating interrelational state- } \\
\text { ments based on defined concepts }\end{array}$ & $\begin{array}{l}\text { - Concepts placed in relation to each other } \\
\text { Relationship statements from tentative state- } \\
\text { inents }\end{array}$ \\
\hline & $\begin{array}{l}\text { Description of model (central con- } \\
\text { cepts, statements, structure and } \\
\text { process) }\end{array}$ & $\begin{array}{l}\text { - } \\
\text { - }\end{array}$ \\
\hline Phase III & $\begin{array}{l}\text { Descriptive deductions and recom- } \\
\text { mendations for applicability }\end{array}$ & $\begin{array}{l}\text { Deduction and inference of guidelines for } \\
\text { operationalisation } \\
\text { - } \quad \text { Literature control } \\
\text { Conversations with experts in theory genera- } \\
\text { tion }\end{array}$ \\
\hline
\end{tabular}

1994: 15).

Methodologically the researcher followed the functional approach (Botes, 1991: 19). According to the functional approach, knowledge is not generated through research for knowledge sakes only, but to be applied to psychiatric nursing practice to provide quality care to patients, and in this case, the HIV reactive patient in rural Malawi.

\section{Research design and method}

The research utilised an exploratory and descriptive, qualitative, contextual and theory generative design. According to Chinn and Kramer (1991: 79 - 145) practice and research are linked and interdependent and should contribute to practice. This research was theory generative in that results of all the fieldwork serve as a basis for generating a model for care counselling in rural Malawi.

To illustrate the course of the research process, a flow diagram was utilised. See Table 1.

The model for care-counselling HIV reactive patients in rural Malawi was generated by utilising the method of theory generation of Chinn and Kramer (1991: 79 - 105), while also em- ploying the approaches of Dickoff, James and Wiendenbach (1986: 429-451), Copi (1986: 157-161), Wilson (1987) and Walker and Avant (1988: 19-194) at different stages of theory development. The following steps were followed in generating the model:

- Identification and definition of central concepts of the model. These concepts were identified from the results obtained in phase 1. The survey list of Dickoff, James and Wiedenbach (1968: 425 - 431) was utilised to direct this process of concept identification and classification. The concept "enable" was identified as a major concept for a model for care-counselling in rural Malawi. This concept was clarified within the unique context of the model by analysing it according to the three-step method of Wilson (1987: 3-10). A dictionary of general definitions, as well as a subject definition of the concept "enable" was written.

- Thereafter, a model case was formulated that would best explain the meaning of the concept "enable" in a psychiatric nursing practice situation. This highlighted the essential characteristics of the concept.

- Conceptual statements, upon which the model was based, were clarified.

- Relationship statements between concepts were for- 
mulated.

Four independent experts subjected the generated model to evaluation. The evaluation was carried out by applying the criteria for the critical reflection of a nursing theory (Chinn and Kramer, 1991: 130-132).

Throughout the research, the researcher utilised different logical reasoning strategies in order to enable the researcher to generate the model, namely: Analysis (Walker \& Avant, 1988:24); Synthesis (Walker \& Avant, 1988:25); Derivation (Walker \& Avant, 1988:25); Inductive reasoning (Bandman \& Bandman, 1988:257); Deductive reasoning (Bandman \& Bandman, 1988: 183-187); Hypothetical-deductive (Bandman \& Bandman, 1988:129-131).

\section{Reliability and validity/ trustworthiness}

Reliability and validity were ensured by means of establishing trustworthiness. Guba and Lincoln's (1985: 290-300) model for trustworthiness of qualitative research was employed. It is based on the identification of four strategies for ensuring trustworthiness used in research studies, namely: (a) truth-value, (b) applicability, (c) consistency and (d) neutrality.

\section{- $\quad$ Truth value}

Truth-value determines whether the researcher has established confidence in the truth of the findings for the subjects or informants and the context in which the research was undertaken. In qualitative research truth-value is usually obtained from the discovery of human experiences as they are lived and perceived by informants.

In this research, truth-value was obtained by using the strategy of credibility and the following criteria, namely triangulation by using two or more theories, data sources, methods and investigators in the study of the phenomenon. Peer examination was done through discussions with Doctorally-prepared experts who are advanced practitioners in psychiatric nursing and experienced in research methods. They critically evaluated the generated model and guidelines using certain criteria. Establishing the authority of the researcher - the researcher has done a course in research methodology and a Master's degree in psychiatric nursing. Establishment of structural coherence was ensured by focusing on enablement and a model for care-counselling and using the framework of the Nursing for the Whole Person Theory. Establishment of referential adequacy was done by means of subject literature definitions of central concepts, and the use of a literature control.

\section{- Applicability}

Applicability refers to the degree to which the findings can be applied to other contexts and settings or with other groups; it is the ability to generalise from the findings to larger populations. The strategy of transferability was applied using the following criteria: A nominated sample was used by means of purposively selecting participants. The sample was compared to demographic data, i.e. to the findings of other researchers and what been documented in the literature. A dense description of the methodology as well as the generated model was provided.

\section{- Consistency}

Consistency considers whether the finding will be consistent if the inquiry were replicated with the same subjects or in a similar context. Since the qualitative setting may be complicated by extraneous and unexpected variables, consistency was defined in terms of dependability, which implies traceable variability, that is, variability that can be ascribed to identifiable sources. The strategy of dependability was utilised. Dependability was ensured through the following criteria: A dependability audit was done by an independent coder. Stepwise

\section{Figure 1 : Assessment of coping ability of patient within household}

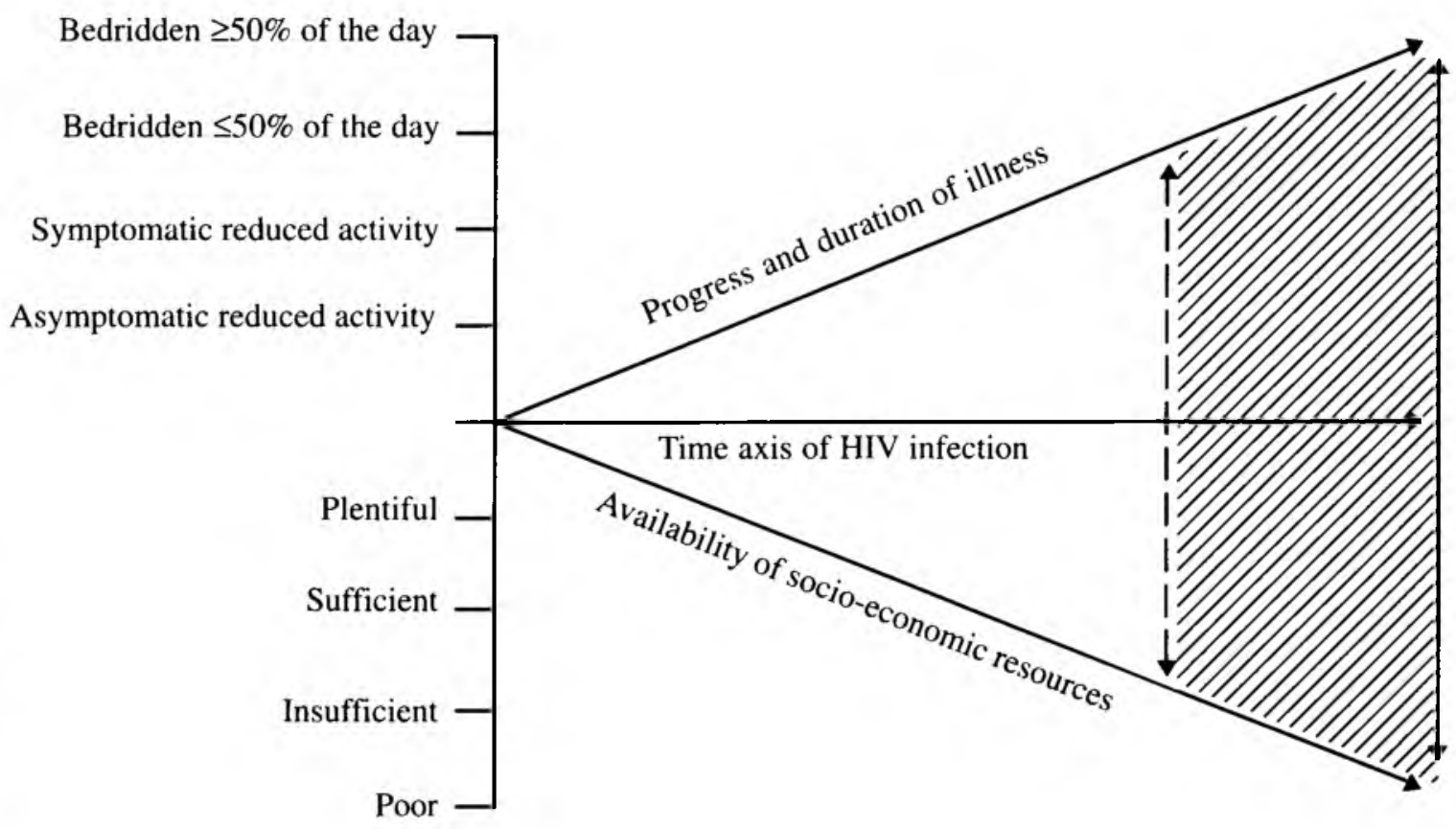


replication was done with the supervision of an advanced researcher throughout the course of the research. The other criteria of triangulation and peer examination were discussed under credibility.

\section{- Neutrality}

Neutrality entails freedom from bias in the research procedures and results and refers to the degree to which the findings are a function solely of the informants and conditions of the research and not of other biases, motivations and perspectives. The strategy of confirmability was utilised and this was ensured by the following criteria: confirmability audit and triangulation as discussed previously. The researcher employed reflexivity in analysing the model case in order to identify concepts from which the creation of conceptual meaning was done; and also in doing a literature control in order to identify ideas that would be used in deductive reasoning to compile guidelines.

The final conceptualised model will now be discussed:

\section{Description of the model for care-counselling of HIV reactive patients in rural Malawi}

Figure 1 depicts the final generated model, which will serve as basis for the description of this model . Concepts applicable to this model were analysed, defined and classified from the fieldwork.

Model generation followed the steps of: Determination of assumptions; identification, classification, and definition of concepts; placement of the concepts in relationship with each other; description of the structure and process of the model; description of the goal and boundaries; and evaluation.

The central concept of this model was "enable", and a conceptual definition, according to Copi's (1986: 157) principles was as follows:

"To enable refers to the process of facilitation where the counsellor as negotiator strengthens the HIV reactive patients who narrates his/her illness experience, which allows the counsellor to identify needs and resources and organise means and opportunities for the participating patient/household to plan and to be mobilised sufficiently to meet the identified internal and external needs of the patient within the household and caring environment"

The analysis of the data showed that the patients were already in an advanced stage of the HIV infection once the diagnosis was made. The family was seen as an essential support structure for the patients but families felt burdened and unable to deal with the impact of AIDS on the household. The longer the duration of the illness, the more helpless the patients and households were to cope with the impact of the illness. Poor resources and high stigmatisation increased the inability of the patients and households taking in consideration the mentioned factors. (Refer to Part I of these articles)

Results of the study showed that the majority of the patients are only diagnosed to be HIV infected when they are already bedridden to some degree. Patients tend to be from an environment where socio-economic resources are insufficient or poor which in combination with the severity of the illness leads to greater inability to deal with the results of the infection.

From the analysis of the data it can be deducted that the inability to cope with the impact of AIDS is the main concern of the patient and the care givers. If inability to cope it the main problem then the goal of counselling will be to enable the patient to cope with the impact of HIV/AIDS within the household and community. Enable therefore became the main concept of the model.

The above central concept, as well as other associated concepts that form the model, were identified and defined, utilising Dickoff, James \& Wiedenbachs' (1968:420) approach and these will now be stated, before the process and evaluation of the model are discussed. By utilising Dickoff, James \& Wiedenbachs' (1968: 423) survey list, the following concepts for the model were derived:

\section{- Agent: Counsellor}

The counsellor is a person who has received training in HIV/ AIDS related matters and counselling and who is affiliated to the formal health sector by being involved with HIV infected patients part-time or full time. The counsellor takes on the role of negotiator. Negotiation is conceptualised as a bargaining process between the counsellor, the patient and household. The focus of negotiation is on the management of illness in order to create maximum relative normality in the face of incapacitation (Sliep, 1994:167).

\section{- Recipient: Patient}

The patient is any individual, family or community who is in interaction with the formal health sector. For the purpose of the study the patient is an HIV reactive Malawi who is seen as part of a family and part of a community. The patient takes on the role of narrator. This enables the patients to tell his/her story to give coherence to distinctive events and the longterm course of suffering. The narrative forms an important channel of communication where the content of the story expresses meaning for the patient. Through this process the counsellor forms a rapport with the patient and is able to negotiate an acceptable therapeutic approach (Sliep, 1994: 166$167 ; 178)$.

\section{- Process}

The counsellor strengthens, negotiates and organises means and opportunity in a process of facilitation, caring and mobilisation during which the patient participates, narrates and plans to meet identified internal and external needs of patient within household (Sliep, 1994:177;179).

\section{- CARE as concept}

The acronym CARE is one method of illustrating the importance of community involvement in the process of enablement. $\mathrm{C}=$ Community $\mathrm{A}=$ Action $; \mathrm{R}=$ Rends $; \mathrm{E}=$ Enablement. On the basis of a lot of feedback from counsellors in the field (Sliep, 1994: 179) it was decided to call the model the CARE Counselling Model.

\section{- Context}

The context of the model is counselling, co-ordinated from the 
formal health sector within a rural community in Malawi, with poor resources and patients with an advanced stage of HIV infection. Malawi's adhere to an African worldview where group identification and hierarchical structures are important. Patients have received limited formal education and need guidance in problem solving during the counselling process. Patients are already in an advanced stage of the illness when they are diagnosed with HIV infection. Diagnoses happens by request of a medical person and not by request of the patients (Sliep, 1994: 186).

\section{Terminus (goal)}

To enable the HIV reactive patient within the household in a rural community in Malawi.

Associated concepts:

\section{Counselling}

Counselling is a dynamic interactive process involving a patient (individual, family or community) and a counsellor. The approach is problem based and aims towards effective decision making. In relation to HIV/AIDS the counselling process assist patients in expressing and ventilating their feelings, fears and concerns, and explores various behavioural practices which may put them at risk of HIV infection. Informed consent is obtained and results are given on request.

\section{Mental Health}

Mental health is an integral part of health (physical, psychological, social and spiritual) and is a dynamic process where psychological processes of the individual, family and community are brought into harmonious interaction with the internal and external environment.

Self: The individual as an object to his/her own reflective consciousness, the agent in his/her own activities, the subject of his/her won feelings, and the possessor of faculties and character.

Culture: Culture is a field of action, which contents range from objects and use by human beings to institutions, ideas and myths. Being an action field, culture offers possibilities of, but by the same token stipulates conditions for, action; it circumscribes conditions that can be reached by a certain means, but also establishes limits for correct, possible and deviant action. As an action field, culture not only induces and controls action, but is also continuously transformed by it.

Community: A society of people is a group who has common rights or privileges, or common interests, civil, political or ecclesiastical; or who live under the same laws and regulations. For the purpose of this study a community will also include geographical boundaries.

Family: The collective body of persons living in one or more houses next to each other under one household head.

Care giver: This is the person who takes primary responsibility in the family for the needs of the patient known as the guardian in Malawi.

Environment: Environment includes both the internal and external world of the patient, family and community as well as the counsellor and health sector. The environment encompasses the culture, circumstances and influences under which the counsellor and patient interact with one another

Relationship statements were formulated, which serve to put concepts in relation with one another. The following statements may relate the concepts above to one another:

Relationship statements of the model:

- A process of enablement takes place between the counsellor, the patient and possibly also the house hold the patient comes from.

- The relationship between the counsellor and the patient remains the primary relationship. The household is involved in the counselling process by request of the patient that is encouraged by the counsellor.

- $\quad$ The sicker the patient and the greater the lack of resources, the greater the inability of the patient and the household to deal with the impact of AIDS.

- $\quad$ The greater the inability of the patient and the household to deal with the impact of AIDS, the more active the role of the counsellor to enable the patient within the household and the community.

- The enablement process starts from the moment when help is requested until after the death of the patient when the affected household then becomes the focus of enablement.

- Enablement of the patient and household has an enabling effect on the counsellor.

To explain the above, the counsellor fulfils the role of facilitator to enable the HIV reactive patient within the household and community. The counsellor is in interaction with the patient, family and community through a process of narration and negotiation over a period of time. Enablement fully takes place if all three phases of the counselling process are actively participated in by the patient, the household and the community. To enable refers to the process of facilitation where the counsellor as negotiator strengthens the HIV reactive patient who narrates his/her illness experience which allows the counsellor to identify needs and resources and organise means and opportunities for the participating patient/household to plan and to be mobilised sufficiently to meet the identified internal and external needs of the patient within the household and caring environment.

The process of enablement addresses both the internal and external needs of the HIV reactive patient and also has an enabling effect on the counsellor who is now addressing the needs expressed by the patient and therefore feels more functional.

\section{THE PROCESS description of THE MODEL}

The overall goal of the model for care-counselling of HIV reactive patients in rural Malawi is to enable the HIV reactive patient within the household and community.

The process of the CARE counselling model takes place in three distinct phases, namely pre-test counselling, post-test counselling and community care. If any of these stages is not followed through completely the process of enablement will not develop to its full potential. 
The counsellor has a hierarchical relationship with the patient according to the traditional structures the patient is used to. The time frame used for counselling is episodic with long sessions (if the condition of the patient allows it) in order to possibly establish a process over time. The time focus is the present, taking the immediate past into consideration for the purpose of history taking, and the immediate future for the purpose of forming a plan of action. As a role model, the counsellor is caring and negotiating. The counselling is person centred and placed within a household and community context.

Analysis of the data revealed that counsellors tend to go over into an education mode when they feel insecure and giving information thus dominates the session. It is therefore suggested that HIV/AIDS information be kept to a minimum during the session. The patient is exposed more HIV/AIDS information in an unthreatening environment in a group context, where the possibilities exist to enter into dialogue with the person offering the information.

In agreement and for the protection of the patient the counselling is started with the patient alone. Permission is sought from the patient as early as possible to include at least one other significant member of the household. Pre-test counselling, as every other phase of counselling, could consist of more than one session in order to achieve the necessary objectives. During the process of counselling the counsellor will attempt to increase shared confidentiality with as many people the patient is comfortable with in order to achieve supportive interactions. All phases of the counselling, namely pre-test counselling, post-test counselling and community care, are important for the enablement of the patient within the household.

\section{- $\quad$ Pre-test counselling}

During the pre-test counselling there is mutual interaction between the counsellor and the patient during which rapport is built with the patient. The counsellor facilitates a process of narration by the patient. The patient narrates his/her personal story of the illness, which is an unthreatening subject for the patient to discuss. The patient is usually focused on the pain and other uncomfortable symptoms of the HIV-related illness that facilitates narration. Through the process of narration the counsellor comes to understand the meaning of illness of the patient as well as the consequences the illness has on the patient's life and social world. Information is elicited regarding the impact of the illness on the household, work, income and other important aspects within the patient's social context. Social support networks and coping strategies are identified, including the use of traditional medicines. The information obtained can be used to assess the coping ability of the patient and the household to deal with the impact of AIDS.

\section{- Post-test counselling}

During post-test counselling the results of the HIV test are given to the patient. The test results, in the context of this research study, are mostly HIV reactive. The patient culturally has difficulty showing emotions and tends to go over into a passive acceptance mode, as was explained during the analysis of the results. The assessment made during the pre-test counselling is presented to the patient and the household members and options and possibilities are explored together. The counsellor takes on the role of negotiator towards the patient and the involved household member(s) in order to organise means and opportunities, which include the mobilisation of resources that will strengthen the patient within the household. The tasks concerning the care of the patient and dealing with the implications of a sick household member are negotiated with the household members present at the counselling session. The identified social networks and coping strategies are actively mobilised through further discussions and open communication where the patient is still encouraged to narrate ideas and experiences.

\section{- Community care}

The patient is followed up in the community where the counsellor aims to create supportive interaction between the household and community members. Initially the patient as narrator was the main actor, while a complete assessment was conducted; then the counsellor became the main actor, in terms of negotiating the acceptance and support of the patient and the sharing of the tasks involved in caring for a dying patient. During community care every person is regarded as an important actor and contributor to create supportive interaction. Ideas and questions are encouraged from all members involved in the life of the patient; this is done with the permission of the patient that is also culturally congruent with how illness is managed in the community. The counsellor facilitates open communication and active participation from all members to enhance a caring environment and the process of enablement.

\section{Evaluation of this model}

Five independent experts subjected the model to evaluation. They utilised the criteria for critical reflection of a nursing theory according to Chinn \& Kramer (1991:130-132).

\section{Simplicity}

Simplicity of a theory means that the number of elements within each descriptive category, particularly concepts and their interrelationships, are minimal. All evaluators found the process of the model when looking at the different phases.

\section{- Generality}

Generality of a theory refers to its breath of scope, which is reflected by the scope of the concepts and purpose within the theory. All evaluators agreed that although the model was developed for a specific context, namely rural Malawi, it would be possible to use the model in other contexts.

\section{- Clarity}

Clarity refers to how well the theory (model) can be understood and how consistently the ideas are conceptualised. Unclear concepts were identified and recontextualised within the model.

\section{- Accessibility}

Accessibility addresses the extent to which empirical indicators can be identified for concepts within the theory, and also how attainable the projected outcomes of the theory are. The empirical grounding of this model was clear and any repetition was omitted. 


\section{- Importance}

Importance of the theory addresses the extent to which the theory leads to valued nursing goals in practice, in research and in education. Every evaluator agreed that the model is very useful and meaningful and recommended that it be implemented as widely as possible.

\section{Guidelines for enabling the HIV reactive patient in rural Malawi}

The objectives, strategies and activities that are suggested for each phase of counselling are guidelines that are dynamic in nature. There is further a clear overlap of the phases and some aspects may have to be repeated in any of the other sessions. Each stage of the counselling process could entail more than session, which is encouraged.

This article will refer to the guidelines in short, but the reader is referred to Sliep (1994: 197-204) for a more comprehensive description of proposed activities to reach the objectives of each stage of counselling.

\section{- Pre-test counselling}

\section{Objectives}

- Establish a rapport with the patient and collect sociodemographic information.

- Conduct a comprehensive need assessment. including physical, psychological, social and spiritual aspects such as the following:

- assessment of economic status of the pa tient within the household

- $\quad$ establish duration and severity of illness

- $\quad$ elicit meaning of illness experience for the patient

identify possible resources: economic, social and spiritual assess ability of patient within household to cope with impact of AIDS

- $\quad$ Prepare patient for HIV test and acquire informed consent to have the test done

- $\quad$ Encourage and assist patient to involve at least one other significant person in the patient's life in the counselling process to create shared confidentiality

- Create an environment that makes it possible for the patients to improve his/her knowledge of HIV/AIDS

\section{Strategy used to obtain the objectives}

Counsellor encourages patient to narrate personal story of illness during pre-test counselling session. Where necessary, use can be made of direct questioning techniques to encourage patients to participate actively.

\section{- Post-test counselling} Objectives

- $\quad$ Prepare the patient for HIV test results:

provide test results

- $\quad$ provide psychosocial and emotional support

- $\quad$ Present plan of action based on needs assessment done during pre-test counselling
- $\quad$ Explore options of each identified need taking a negotiating approach

- $\quad$ Encourage open communication about feelings and problems between the household members and the patient

- Limit HIV transmission risk by providing information and teach skills to manage patient

\section{Strategy}

The counsellor takes on the role of negotiator that is more within the cultural tradition. The counsellor negotiates with the patient, the family and the community in order to mobilise support and assistance and prevent a sense of hopelessness as well as to encourage maximal participation in everyday life. Integration of as many people as possible in the counselling process forms part of the strategy.

\section{- Community care}

\section{Objectives}

- Integrate patient within the household and community

- Mobilise resources for the patient/household

- Integrate patient, household and community into the Primary Health Care programme including homebased care, income-generating activities and orphan care

- $\quad$ Maximise support of the patient within household by facilitating open communication and decreasing stigmatisation of AIDS in the community

- Encourage community participation to enhance a caring environment

\section{Strategy}

Relationship linkages within the extended family and the community allow for spontaneous interweaving of information, accountability and responsibility from the individual to the group and from the group to the individual. Mobilise the community to ensure supportive interaction between houpehold and community members. Shared confidentiality and shared problem ownership could enhance support within the community. Use local wisdom for active participation in problem solving. Continue to enable the household after the death of the patient if necessary.

\section{Conclusion}

In this article a care-counselling model for HIV reactive patients in rural Malawi was described by utilising the final conceptual and theoretical framework. The process and structure was discussed and finally, the model was evaluated by means of specific criteria.

The model proposes that the patient take on the role of narrator while the counsellor negotiates a caring environment among household members and encourages supportive interaction in the community.

"Umodzi ndi chipulumutso"

Chichewa proverb translated meaning

"Togetherness brings hope" 


\section{References}

AIDS SECRETARIAT (AIDS CONTROL PROGRAMME), Ministry of Health Malawi 1994: HIV/AIDS Annual Report, Lilongwe, Malawi.

AIDS CONTROL PROGRAMME, Ministry of Health Malawi 1992: HIV/AIDS Counselling Training Manual, Lilongwe, Malawi.

BANDMAN, EL \& BANDMAN, B 1988: Critical thinking in Nursing. Norwalk: Appleton \& Lange.

BOTES, AC 1991: A functional approach in Nursing. Curationis. 14(1): 19-23.

CHINN, PL \& KRAMER, MK 1991: Theory and nursing systematic approach. St. Louis: CV Mosby.

COPI, IM 1986: Informal logic. New York: MacMillan.

DICKOFF, J; JAMES, P \& WIEDENBACH, E 1968: Theory in a practice discipline. Part I. Practice orientated theory. Nursing Research. 17(5), Sept. - Oct. 1968: 415 - 435.

GUBA, E 1981: Criteria for assessing the trustworthiness of naturalistic inquiries. Educational Resources Information Centre Annual Review Paper. 29, 1981: 75 -91.

KERLINGER, FN 1986: Foundation of behavioural research. New York: Rinehart and Winston Inc.

LINCOLN, YS \& GUBA, EA 1985: Naturalistic inquiry. Beverly Hills, CA: Sage.

ORAL ROBERTS UNIVERSITY ANNA VAUGHN SCHOOL OF NURSING, 1990: Baccalaureate and Higher Degree Programs, Volume I - Narrative by Criteria. Tulsa, Oklahoma: Oral Roberts University Anna Vaughn School of Nursing: 13, 136$139,142$.

SLIEP, Y 1994: Care Counselling Model for AIDS patients in rural Malawi: (Unpublished DCur thesis, RAU)

WALKER, L \& AVANT, K 1988: Strategies for theory construction in nursing, Second Edition. Norwalk: Appleton \& Lange.

WILSON, HS 1987: Research in nursing, Second Edition. Redwood City, California: Addison-Wesley. 